\title{
Anticonvulsant drugs effects on sex hormone levels and sexual function in men with epilepsy
}

\author{
Mehrdokht Mazdeh', Mahzad Heidari², Mohammad Taheri*,3(iD) \& Soudeh \\ Ghafouri-Fard**,4 (iD) \\ ${ }^{1}$ Neurophysiology Research Center, Hamadan University of Medical Sciences, Hamadan, Iran \\ ${ }^{2}$ Department of Neurology, Hamadan University of Medical Sciences, Hamadan, Iran \\ ${ }^{3}$ Urogenital Stem Cell Research Center, Shahid Beheshti University of Medical Sciences, Tehran, Iran \\ ${ }^{4}$ Department of Medical Genetics, Shahid Beheshti University of Medical Sciences, Tehran, Iran \\ *Authors for correspondence: Tel.: \& Fax: +982123972572; mohammad_823@yahoo.com \\ **Authors for correspondence: s.ghafourifard@sbmu.ac.ir
}

\begin{abstract}
Aim: Sexual dysfunction is common in patients with epilepsy. The aim of this quasi-experimental study was to evaluate the effect of anticonvulsant drugs on hormonal and sexual function in men with epilepsy. Methods: Newly diagnosed patients with epilepsy were divided into three groups of 23 subjects who received monotherapy with sodium valproate, carbamazepine and levetiracetam, respectively. Findings: At the beginning of the study, sexual function and sexual hormones were comparable between the control group and the patient group $(p>0.05)$. After 6 months of treatment, patients who received sodium valproate had the weakest sexual function. Conclusion: According to the present findings, levetiracetam appears to be a good choice for sexually active patients compared with other conventional anticonvulsants.
\end{abstract}

First draft submitted: 12 December 2019; Accepted for publication: 5 February 2020; Published online: 18 March 2020

\section{Keywords: antiepileptic drugs $\bullet$ epilepsy $\bullet$ sexual function}

Epilepsy refers to a common chronic neurological condition associated with considerable morbidity and mortality [1]. An epileptic seizure is a type of anomalous neuronal firing which can be classified into two classes: generalized and focal. While the former is originated from the neuronal network of a certain region of one cerebral hemisphere, the latter is initiated from bilateral dispersed neuronal networks [2]. According to epidemiological and clinical evidence, epilepsy is accompanied by abnormal sexual function due to both pathogenic events of epilepsy and the use of antiepileptic drugs (AEDs). A significant number of epileptic men experience sexual dysfunction. Moreover, sexual dysfunction has been reported as a common side effect of AEDs [3]. A significant number of epileptic men experience sexual dysfunction $[4,5]$. The reported symptoms indicated that AEDs could influence erectile function, sexual desire and ejaculatory function. Traditional AEDs such as sodium valproate have been associated with decreased sexual desire [6]. Epilepsy also influences the reproductive system-related endocrine functions [3]. Some investigations have also suggested disturbances in the function of gonadotropin-releasing hormone pulse generator in the temporal lobe epilepsy [7]. Dysregulation of the hypothalamic-pituitary-adrenal axis has also been introduced as one of the underlying mechanisms of AED-related sexual dysfunction [8] especially for carbamazepine, phenytoin and sodium valproate [9]. Several liver enzyme-inducing AEDs including carbamazepine can enhance the serum levels of sex hormone-binding globulin (SHBG), hence decreasing the testosterone bioactivity [7]. Administration of sodium valproate and carbamazepine has been also associated with dysregulation of sex hormones, sexual dysfunction and changes in semen parameters [10]. On the other hand, AEDs such as oxcarbazepine, lamotrigine and levetiracetam have been reported to enhance the sexual function [6]. In this regard, the current quasi-experimental study is aimed to compare the effects of sodium valproate, carbamazepine and levetiracetam on plasma levels sex hormones and sexual function of naïve epileptic patients. The basis for the selection of these AEDs was their wide administration in epileptic patients among the assessed population.

Future Medicine 


\begin{tabular}{|c|c|c|c|c|c|}
\hline Groups & Sodium valproate & Carbamazepine & Levetiracetam & Control & p-value \\
\hline Sexual function & $40.8 \pm 14.9$ & $52.2 \pm 7.8$ & $57.1 \pm 5.3$ & $58.2 \pm 6.6$ & 0.001 \\
\hline Erection (per month) & $17.3 \pm 6.8$ & $22.1 \pm 3.6$ & $23.7 \pm 2.5$ & $24.2 \pm 2.9$ & 0.001 \\
\hline Orgasm (per month) & $6.7 \pm 2.2$ & $7.3 \pm 1.1$ & $8.1 \pm 1.1$ & $8.0 \pm 1.1$ & 0.013 \\
\hline Intercourse satisfaction & $5.0 \pm 2.2$ & $6.4 \pm 1.2$ & $7.1 \pm 0.9$ & $7.3 \pm 1.1$ & 0.001 \\
\hline Overall satisfaction & $7.8 \pm 3.9$ & $11.3 \pm 3.2$ & $12.3 \pm 1.5$ & $12.9 \pm 2.8$ & 0.001 \\
\hline Sexual desire & $4.5 \pm 1.7$ & $5.6 \pm 1.5$ & $6.0 \pm 1.7$ & $6.4 \pm 0.9$ & 0.128 \\
\hline
\end{tabular}

\section{Patients \& methods}

\section{Study design}

This quasi-experimental study was conducted in 2018 in Farshchian Hospital and affiliated clinics of Hamadan University of Medical Sciences. The study protocol was approved by ethical committee of Hamadan University of Medical Sciences. Informed consent forms were obtained from all study participants. Epileptic patients between the ages of 18 and 50 years, who gave consent and were married and sexually active, were included in the study. The 'consecutive sampling' technique was used for recruitment of study participants. Appropriate sample size for the study was calculated based on the results of Heroz et al. study by using STATA software [11] considering $\alpha$ of 0.05 and study power of $80 \%$ [12]. Finally, the study was conducted on 69 male subjects diagnosed with generalized or focal epilepsy and 23 healthy male subjects. Diagnosis of epilepsy was based on brain MRI and EEG findings. Control subjects were age-matched with patients. Patients with epilepsy were divided into three groups of 23 subjects who further received monotherapy with sodium valproate, carbamazepine and levetiracetam, respectively.

Exclusion criteria were the presence of endocrine disorders such as diabetes mellitus or thyroid or hypothalamohypophysial dysfunction, major depressive disorder or other psychiatric disorder, anatomical defects in testes including varicocele or spermatocele cyst, alcohol abuse, history of hormone therapy and any other systemic disorder.

Plasma levels of luteinizing hormone (LH), follicle-stimulating hormone (FSH), free testosterone and SHBG were assessed in all study participants at baseline and 6 months later. Sexual function was evaluated by the International Index of Erectile Function self-report instrument [13], Persian version [14].

\section{Statistical methods}

Data were analyzed using SPSS v. 16 (IBM Corp, IL, USA). p-values lower than 0.05 were regarded as significant. Quantitative variables were designated using mean and standard deviation (SD), while categorical variables were described as ratios or percentages. Categorical variables were compared using $\chi^{2}$ test. t-test or nonparametric equivalents were used for comparison of quantitative variables.

\section{Results}

General data of study participants

The current study was conducted on 69 newly diagnosed epileptic male patients and 23 age-matched male control subjects. Supplementary Table 1 shows demographic data of study participants. Fifty-three (76.8\%) patients had generalized epilepsy and 16 (23.2\%) had focal epilepsy.

\section{Sexual function}

Assessment of sexual function by International Index of Erectile Function self-report instrument showed no significant difference in any parameter between study subgroups at the beginning of the study (Supplementary Table 2).

After 6 months, scores were significantly decreased in patients compared with controls. The worse sexual function scores were seen in patients treated with sodium valproate, while levetiracetam had the least negative effects. All sexual performance criteria except for sexual desire were lower in patients receiving sodium valproate compared with control group $(\mathrm{p}<0.05)$ (Table 1). Comparison of mean values showed significant difference between sodium valproate and levetiracetam in all assessed domains (Table 2). 
Table 2. Comparison of sexual function parameters between study subgroups ( $p$-values are shown).

\begin{tabular}{|llll|}
\hline Groups & Sodium valproate vs carbamazepine & Sodium valproate vs levetiracetam & Levetiracetam vs carbamazepine \\
\hline Sexual function & 0.002 & 0.001 & 0.015 \\
\hline Erection (per month) & 0.004 & 0.001 & 0.089 \\
\hline Orgasm (per month) & 0.273 & 0.007 & 0.008 \\
\hline Intercourse satisfaction & 0.014 & 0.001 & 0.074 \\
\hline Overall satisfaction & 0.001 & 0.001 & 0.164 \\
\hline Sexual desire & 0.095 & 0.025 & 0.363 \\
\hline
\end{tabular}

Table 3. Frequency of erectile dysfunction in study subgroups at the beginning of the study.

\begin{tabular}{|c|c|c|c|}
\hline Group & $\begin{array}{l}\text { Erectile dysfunction } \\
\text { Number (\%) }\end{array}$ & $\begin{array}{l}\text { Normal erectile function } \\
\text { Number (\%) }\end{array}$ & p-value ${ }^{\dagger}$ \\
\hline Sodium valproate & $17(30.9)$ & $6(16.2)$ & 0.446 \\
\hline Carbamazepine & $13(23.6)$ & $10(27.0)$ & \\
\hline Levetiracetam & $12(21.8)$ & $11(29.7)$ & \\
\hline Control & $13(23.6)$ & $10(27.1)$ & \\
\hline Sum & $55(100)$ & $37(100)$ & \\
\hline
\end{tabular}

\begin{tabular}{|c|c|c|c|}
\hline Group & $\begin{array}{l}\text { Erectile dysfunction } \\
\text { Number (\%) }\end{array}$ & $\begin{array}{l}\text { Normal erectile function } \\
\text { Number (\%) }\end{array}$ & $p$-value ${ }^{\dagger}$ \\
\hline Sodium valproate & $20(31.7)$ & $3(10.3)$ & 0.029 \\
\hline Carbamazepine & $18(28.6)$ & $5(17.2)$ & \\
\hline Levetiracetam & $12(19.1)$ & $11(37.9)$ & \\
\hline Control & $13(20.6)$ & $10(34.5)$ & \\
\hline Sum & $63(100)$ & $29(100)$ & \\
\hline
\end{tabular}

${ }^{\dagger} p$-value is calculated using $\chi^{2}$ test.

Table 5. Plasma levels of luteinizing hormone, follicle-stimulating hormone, sex hormone-binding globulin and testosterone (mean \pm standard deviation) in study subgroups at the beginning of the study.

\begin{tabular}{|llllll}
\hline Hormones & Sodium valproate & Carbamazepine & Levetiracetam & Control & $5.3 \pm 1.8$ \\
\hline LH (IU/l) & $4.7 \pm 1.4$ & $6.4 \pm 1.3$ & $4.9 \pm 1.1$ & 0.683 & $8.1 \pm 1.2$ \\
\hline FSH (IU/I) & $8.30 \pm 0.8$ & $8.4 \pm 0.9$ & $8.7 \pm 0.6$ & 5.409 & $51.0 \pm 7.5$ \\
\hline SHBG (IU/l) & $54.1 \pm 5.7$ & $51.3 \pm 8.3$ & $50.4 \pm 6.7$ & 6.309 & $817.1 \pm 206.1$ \\
\hline Testosterone (ng/dl) & $677.5 \pm 306.4$ & $720.5 \pm 302.6$ & $691.1 \pm 327.8$ & 0.448 & \\
\hline
\end{tabular}

FSH: Follicle-stimulating hormone; LH: Luteinizing hormone; SHBG: Sex hormone-binding globulin.

Erectile dysfunction has been detected in $59.8 \%$ of study participants at the beginning of the study with no significant intergroup differences (Table 3).

At the end of the study, $68.5 \%$ of study participants reported erectile dysfunction. Frequency of erectile dysfunction was significantly higher in patients receiving sodium valproate or carbamazepine compared with control group (Table 4). The difference between sodium valproate and levetiracetam groups was statistically significant $(p=0.023)$. However, no significant differences were found in erectile function between carbamazepine and sodium valproate $(\mathrm{p}=0.350)$ or between carbamazepine and levetiracetam $(\mathrm{p}=0.120)$.

Sex hormone levels

There was no significant difference in plasma levels of LH, FSH, SHBG and testosterone between-study groups (Table 5). 
Table 6. Plasma levels of luteinizing hormone, follicle-stimulating hormone, sex hormone-binding globulin and testosterone (mean \pm standard deviation) in study subgroups after 6 months.

\begin{tabular}{|llllll}
\hline Hormones & Sodium valproate & Carbamazepine & Levetiracetam & Control & \\
\hline LH (IU/I) & $4.5 \pm 1.8$ & $4.6 \pm 1.2$ & $5.2 \pm 1.9$ & $5.3 \pm 1.8$ & 8.683 \\
\hline FSH (IU/I) & $7.60 \pm 2.1$ & $8.4 \pm 1.1$ & $8.4 \pm 1.1$ & 0.409 \\
\hline SHBG (IU/I) & $46.5 \pm 17.8$ & $45.9 \pm 12.0$ & $55.8 \pm 14.7$ & $51.0 \pm 7.5$ \\
\hline Testosterone (ng/dl) & $522.6 \pm 306.6$ & $600.4 \pm 317.9$ & $754.2 \pm 302.7$ & $817.1 \pm 206.1$ \\
\hline FSH: Follicle-stimulating hormone; LH: Luteinizing hormone; SHBG: Sex hormone-binding globulin. & 0.002 \\
\hline
\end{tabular}

\begin{tabular}{|c|c|c|c|}
\hline Groups & Sodium valproate vs carbamazepine & Sodium valproate vs levetiracetam & Levetiracetam vs carbamazepine \\
\hline $\mathrm{LH}(\mathrm{IU} / \mathrm{I})$ & 0.834 & 0.174 & 0.164 \\
\hline FSH (IU/I) & 0.117 & 0.089 & 0.895 \\
\hline SHBG (IU/I) & 0.894 & 0.060 & 0.019 \\
\hline
\end{tabular}

Table 8. Comparison of hormone levels between patients with focal and generalized epilepsy.

\begin{tabular}{|lll|}
\hline Hormones & Focal epilepsy & Generalized epilepsy \\
\hline LH (IU/I) & $4.9 \pm 2.1$ & $4.6 \pm 1.6$ \\
\hline FSH (IU/I) & $7.9 \pm 2.2$ & $8.2 \pm 1.4$ \\
\hline SHBG (IU/I) & $45.1 \pm 17.2$ & $50.5 \pm 16.5$ \\
\hline Testosterone (ng/dl) & $789.1 \pm 13.9$ & $519.4 \pm 303.1$ \\
\hline FSH: Follicle-stimulating hormone; LH: Luteinizing hormone; SHBG: Sex hormone-binding globulin. & 0.920 \\
\hline
\end{tabular}

After 6 months, no significant difference was detected between subgroups except for lower levels of testosterone in sodium valproate group compared with controls (Table 6).

Intergroup comparisons showed significant differences in SHBG levels between levetiracetam and carbamazepine groups and in testosterone levels between sodium valproate and levetiracetam groups (Table 7).

Comparison of hormone levels between patients with focal and generalized epilepsy revealed higher levels of testosterone in the former group compared with the latter (Table 8). Levels of other hormones were not different between these groups.

\section{Discussion}

In the current study, we compared sex hormone levels and sexual function between epileptic patients who received three different AEDs for a period of 6 months and healthy controls. After 6 months, scores of assessments of sexual function were significantly decreased in patients compared with controls. The worse sexual function scores were seen in patients treated with sodium valproate, while levetiracetam had the least negative effects. All sexual performance criteria except for sexual desire were lower in patients receiving sodium valproate compared with control group. Moreover, comparison of mean values of scores showed significant differences between sodium valproate and levetiracetam in all assessed domains. Our results are in accordance with the results of previous studies which reported high frequency of sexual dysfunction in patients treated with AEDs [6]. Similar to our study, previous studies demonstrated erectile dysfunction, hyposexuality and ejaculatory dysfunction as side effects of AEDs [6]. Dose-related anorgasmia has been also reported in patients receiving oxcarbazepine [15]. Moreover, another AED, topiramate can provoke erectile dysfunction [16]. Thus, AEDs have been recurrently associated with different types of sexual dysfunction phenotypes necessitating a comprehensive assessment of patients receiving these drugs. 
While both sodium valproate and carbamazepine has been associated with decreased libido, levetiracetam has been reported to improve sexual function [6]. Therefore, our results regarding the difference in sexual function between patients receiving sodium valproate and levetiracetam accord the bulk of evidence in this regard.

We detected no significant difference in the levels of sex hormones between study subgroups after 6-month treatment with mentioned AEDs except for lower levels of testosterone in sodium valproate group compared with the controls. Our results regarding lower testosterone levels in sodium valproate group is in accordance with Najafi et al. [17]. Yet, the comparable levels of testosterone between carbamazepine group and control group does not accord with the results of this study [17]. The similar levels of sex hormone in patients treated with levetiracetam and control group is in agreement with the study conducted by Spencer on newly diagnosed epilepsy patients which reported decreased sperm parameters in these patients after treatment with levetiracetam without affecting sex hormone levels [18]. Moreover, our study is in accordance with Svalheim et al. who demonstrated no drug-specific sexual or endocrine side effects in patients treated with levetiracetam [19]. Ceyal et al. also reported no significant change in concentrations of sex hormones after treatment with levetiracetam [20]. In line with these study, Mohamed et al. have shown adverse effects of sodium valproate but not levetiracetam on sex hormones [21].

We detected no significant difference in LH and FSH levels after treatment with any of three mentioned AEDs. Asadi-Pooya et al. also demonstrated no significant alterations in levels of these hormone after a 3-month treatment with carbamazepine [22]. Moreover, Rättyä et al. reported no significant difference in LH or FSH levels between men taking carbamazepine and control men [23]. While Mohamed et al. have demonstrated significant decrease in levels of LH and FSH after treatment with sodium valproate [21], Rättyä et al. showed similar levels of LH between men taking sodium valproate and control men [23]. Thus, data regarding the effects of AEDs on LH and FSH are not consistent.

Intergroup comparisons showed lower SHBG levels in carbamazepine groups compared with levetiracetam group. However, SHBG levels were not different between carbamazepine and sodium valproate groups. Carbamazepine as a liver enzyme inducing AED has been shown to increase SHBG levels and decrease concentrations of biologically active sex hormones [24]. Similar SHBG levels between carbamazepine and sodium valproate groups is in accordance with Najafi et al. study [17]. Harden et al. have shown a significant decrease in level of SHBG after a 4-week treatment period with levetiracetam [25].

We also detected higher testosterone levels levetiracetam group compared with sodium valproate group at the end of the study which is in agreement with the previously reported effect of levetiracetam on enhancing testosterone levels [25].

Comparison of hormone levels between patients with focal and generalized epilepsy revealed higher levels of testosterone in the former group compared with the latter. There is no consensus on the relation between reproductive endocrine disorders and type of epilepsy [26]. However, some investigations reported higher frequency of sexual dysfunction in patients with partial epilepsy [27-29]. Thus, future studies are needed to uncover the underlying mechanism of our observation and its association with sexual function of patients.

\section{Summary points}

- In the current quasi-experimental study, we compared the effect of anticonvulsant drugs on hormonal and sexual function in men with epilepsy.

- After 6 months treatment with antiepileptic drugs, sexual function scores were significantly decreased in patients compared with controls.

- The worse sexual function scores were seen in patients treated with sodium valproate, while levetiracetam had the least negative effects.

- All sexual performance criteria except for sexual desire were lower in patients receiving sodium valproate compared with control group.

- Comparison of mean values showed significant difference between sodium valproate and levetiracetam in all assessed domains.

- No significant difference was detected in levels of sex hormones between subgroups except for lower levels of testosterone in sodium valproate group compared with controls.

- Levetiracetam appears to be a good choice for sexually active patients compared with other conventional anticonvulsants. 


\section{Conclusion}

Taken together, our study supports the results of previous studies regarding the suitability of levetiracetam for treatment of sexually active epileptic male patients. However, the effect of this AED on sperm parameters has not been assessed in our study. The clinical implications of our findings support the influence of AEDs on epileptic patients. Therefore, male epileptic patients should be cautious about the administration of AEDs as they cause sexual dysfunction or abnormalities in sex hormone levels and may lead to abnormal sperm parameters. This point should be taken into account especially for epileptic patients who wish to conceive.

\section{Financial \& competing interests disclosure}

The current study was supported from Hamadan University of Medical Sciences. The authors have no other relevant affiliations or financial involvement with any organization or entity with a financial interest in or financial conflict with the subject matter or materials discussed in the manuscript apart from those disclosed.

No writing assistance was utilized in the production of this manuscript.

Ethical conduct of research

The study protocol was approved by ethical committee of Hamadan University of Medical Sciences. Informed consent forms were obtained from all study participants. The authors state that they have obtained appropriate institutional review board approval or have followed the principles outlined in the Declaration of Helsinki for all human or animal experimental investigations. In addition, for investigations involving human subjects, informed consent has been obtained from the participants involved.

\section{Open access}

This work is licensed under the Attribution-NonCommercial-NoDerivatives 4.0 Unported License. To view a copy of this license, visit http://creativecommons.org/licenses/by-nc-nd/4.0/

\section{References}

Papers of special note have been highlighted as: • of interest; $\bullet \bullet$ of considerable interest

1. Banerjee PN, Filippi D, Allen Hauser W. The descriptive epidemiology of epilepsy-a review. Epilepsy Res. 85(1), 31-45 (2009).

2. Stafstrom CE, Carmant L. Seizures and epilepsy: an overview for neuroscientists. Cold Spring Harb. Perspect. Med. 5(6), (2015).

3. Atif M, Sarwar MR, Scahill S. The relationship between epilepsy and sexual dysfunction: a review of the literature. Springerplus 5(1), 2070 (2016).

- A comprehensive review of sexual abnormalities in epileptic patients.

4. Nikoobakht M, Motamedi M, Orandi A, Meysamie A, Emamzadeh A. Sexual dysfunction in epileptic men. Urol J. 4(2), 111-117 (2007).

-. New insights into the mechanism of sexual abnormalities in epileptic patients.

5. Herzog AG, Seibel MM, Schomer DL, Vaitukaitis JL, Geschwind N. Reproductive endocrine disorders in men with partial seizures of temporal lobe origin. Arch. Neurol. 43(4), 347-350 (1986).

6. Yang Y, Wang X. Sexual dysfunction related to antiepileptic drugs in patients with epilepsy. Exp. Opin. Drug Safety 15(1), 31-42 (2016).

7. Verrotti A, D’Egidio C, Mohn A, Coppola G, Parisi P, Chiarelli F. Antiepileptic drugs, sex hormones, and PCOS. Epilepsia 52(2), 199-211 (2011).

8. Franceschi M, Perego L, Cavagnini F et al. Effects of long-term antiepileptic therapy on the hypothalamic-pituitary axis in man. Epilepsia 25(1), 46-52 (1984).

9. Lambert MV. Seizures, hormones and sexuality. Seizure 10(5), 319-340 (2001).

10. Ocek L, Tarhan H, Uludag FI et al. Evaluation of sex hormones and sperm parameters in male epileptic patients. Acta Neurol. Scand. 137(4), 409-416 (2018).

11. Stata User's Guide: Release 10. StataCorp LP, TX, USA (2007).

12. Heroz AG, Levesque LA, Drislane FW, Ronthal M, Schomer DL. Phenytoin-induced elevation of serum estradiol and reproductive dysfunction in men with epilepsy. Epilepsia 32(4), 550-553 (1991).

13. Rosen RC, Cappelleri JC, Gendrano N, 3rd. The International Index of Erectile Function (IIEF): a state-of-the-science review. Int. J. Impotence Res. 14(4), 226-244 (2002).

14. Pakpour AH, Zeidi IM, Yekaninejad MS, Burri A. Validation of a translated and culturally adapted Iranian version of the International Index of Erectile Function. J. Sex Mar. Ther. 40(6), 541-551 (2014).

15. Calabro RS, Ferlazzo E, Italiano D, Bramanti P. Dose-dependent oxcarbazepine-related anorgasmia. Epilepsy Behav. 17(2), 287-288 (2010). 
16. Calabro RS, Bramanti P, Italiano D, Ferlazzo E. Topiramate-induced erectile dysfunction. Epilepsy Behav. 14(3), 560-561 (2009).

17. Najafi MR, Ansari B, Zare M, Fatehi F, Sonbolestan A. Effects of antiepileptic drugs on sexual function and reproductive hormones of male epileptic patients. Iran. J. Neurol. 11(2), 37-41 (2012).

18. Spencer D. Levetiracetam in men with epilepsy: testosterone is left alone but sperm count is paramount. Epilepsy Curr. 17(2), 99-100 (2017).

19. Svalheim S, Tauboll E, Luef G et al. Differential effects of levetiracetam, carbamazepine, and lamotrigine on reproductive endocrine function in adults. Epilepsy Behav. 16(2), 281-287 (2009).

20. Ceylan M, Yalcin A, Bayraktutan OF, Karabulut I, Sonkaya AR. Effects of levetiracetam monotherapy on sperm parameters and sex hormones: Data from newly diagnosed patients with epilepsy. Seizure 41, 70-74 (2016).

21. Mohamed WS, Nageeb RS, Hashim NA, Omran AA. Effect of valproate versus levetiracetam monotherapy on reproductive functions in newly diagnosed epileptic males. Egypt J. Neurol. Psychiat. Neurosurg. 55(1), 43 (2019).

22. Asadi-Pooya AA, Dabbaghmanesh MH, Ashjazadeh N. Effects of carbamazepine on male reproductive hormones. Med. J. Islam Repub. Iran. 28, 139 (2014).

23. Rättyä J, Turkka J, Pakarinen AJ et al. Reproductive effects of valproate, carbamazepine, and oxcarbazepine in men with epilepsy. Neurology 56(1), 31-36 (2001).

24. Svalheim S, Sveberg L, Mochol M, Taubøll E. Interactions between antiepileptic drugs and hormones. Seizure 28, 12-17 (2015).

25. Harden CL, Nikolov BG, Kandula P, Labar DR, Pannullo S. Effect of levetiracetam on testosterone levels in male patients. Epilepsia 51(11), 2348-2351 (2010).

26. Tauboll E, Sveberg L, Svalheim S. Interactions between hormones and epilepsy. Seizure 28, 3-11 (2015).

27. Quigg M, Kiely JM, Shneker B, Veldhuis JD, Bertram III EH. Interictal and postictal alterations of pulsatile secretions of luteinizing hormone in temporal lobe epilepsy in men. Ann. Neurol. 51(5), 559-566 (2002).

28. Baird AD, Wilson SJ, Bladin PF, Saling MM, Reutens DC. Sexual outcome after epilepsy surgery. Epilepsy Behav. 4(3), $268-278$ (2003).

29. Herzog AG, Coleman AE, Jacobs AR et al. Interictal EEG discharges, reproductive hormones, and menstrual disorders in epilepsy. Ann. Neurol. 54(5), 625-637 (2003). 